Journal of

Cardiology and Vascular Medicine

\title{
Impact of Coronary Artery Disease on Long-Term Cognitive Outcome after Ca- rotid Endarterectomy
}

Akira Nakamizo, MD, PhD ${ }^{1, *}$; Toshiyuki Amano, MD, PhD ${ }^{1}$; Takahiro Kuwashiro, MD, $\mathrm{PhD}^{2}$; Masahiro Yasaka, $\mathrm{MD}, \mathrm{PhD}^{2}$; Yasushi Okada, $\mathrm{MD}, \mathrm{PhD}^{2}$

${ }^{1}$ Department of Neurosurgery, Clinical Research Institute, National Hospital Organization, Kyushu Medical Center ${ }^{2}$ Cerebrovascular Medicine and Neurology, Clinical Research Institute, National Hospital Organization, Kyushu Medical Center *Corresponding author: Akira Nakamizo, MD, PhD, Department of Neurosurgery, Clinical Research Institute, National Hospital Organization, Kyushu Medical Center, 1-8-1 Jigyohama, Chuo-ku, Fukuoka, 810-8563, Japan; Tel:+81-92-8520700Fax:+81-92-846-8485, E-mail:nakamizo@ns.med.kyushu-u.ac.jp

Received Date: June 18, 2019 Accepted Date: July 15, 2019 Published Date: July 17, 2019

Citation: Akira Nakamizo (2019) Impact of Coronary Artery Disease on Long-Term Cognitive Outcome after Carotid Endarterectomy. J Cardio Vasc Med 5: 1-9.

\begin{abstract}
Objective: Carotid stenosis and coronary artery disease (CAD) are associated with cognitive decline, but little is known about the impact of CAD on cognitive outcome after carotid endarterectomy (CEA). The aim of this study was to assess the long-term cognitive outcomes after CEA in patients with CAD and to identify causative risk factors for cognitive decline.

Methods: Among 36 consecutive patients with CAD who had undergone CEA between February 2016 and June 2018, and 45 patients without $\mathrm{CAD}$ who had undergone CEA during the same period, cognitive functions were studied using the Neurobehavioral Cognitive Status Examination (Cognistat) and the Frontal Assessment Battery (FAB). Cognitive decline was defined as both FAB total score below the cutoff value and severe impairment in at least one domain on Cognistat.

Results: Cognitive decline was evident in $27.8 \%$ of patients with CAD at a mean of 7.2 years after CEA, and in $8.9 \%$ of patients without CAD. Comprehension, memory, and conceptualization were significantly impaired in patients with CAD compared to patients without CAD ( $\mathrm{p}=0.0274,0.0392$, and 0.0113 , respectively).In patients with CAD, univariate analysis revealed that deep white matter hyperintensity (DWMH) and chronic kidney disease associated with cognitive decline (odds ratio (OR) 32.2, 95\% confidence interval (CI) 3.20-323.66, $\mathrm{p}=0.0032$; OR 6.11, 95\%CI 1.13-33.19; $\mathrm{p}=0.0360$, respectively).

Conclusions: This result may be useful to better identify individuals at high risk of cognitive decline long after CEA.

Keywords: carotid endarterectomy; coronary artery disease; cognitive decline; deep white matter hyperintensity; chronic kidney disease
\end{abstract}

(C)2019 The Authors. Published by the JScholar under the terms of the Creative Commons Attribution License http://creativecommons.org/licenses/ by $/ 3.0 /$, which permits unrestricted use, provided the original author and source are credited. 


\section{Introduction}

Carotid stenosis and coronary artery disease (CAD) are associated with cognitive decline, as both are phenotypes of atherosclerosis and share common vascular risk factors. Carotid stenosis can cause cognitive decline through hemodynamic and embolic mechanisms. Carotid endarterectomy (CEA) is expected to maintain or improve cognitive function by reversing the pathological conditions, but CEA itself carries risks of cognitive decline due to micro embolism arising during the surgical procedure, hypoperfusion during carotid artery clamping, general anesthesia, and postoperative hyperperfusion [1]. CEA reduced the frequency of microemboli from $25.0 \%$ to $17.9 \%$ at 1 year after surgery[2], suggesting that risk of cognitive decline persists even after CEA. Thus, CEA can cause both cognitive improvements and declines, but whether these complex interactions ultimately result in an improved or deteriorated cognitive function remains unclear [3].

On the other hand, patients with CAD risk of cognitive impairment[4], and cognitive decline is a common complaint amongthesepatients $[5,6]$. However, little is known about the impact of CAD on cognitive outcome after CEA. This study aimed to assess the long-term cognitive outcomes after CEA in patients with $\mathrm{CAD}$ and to identify causative risk factors for cognitive decline.

\section{Methods}

\section{Patients}

Thirty-six patients with CAD who had undergone CEA and were admitted to our institution between February 2016 and June 2018 for periodic follow-up were invited to participate in this study. Clinical data were obtained from patients by retrospective chart review. CAD was confirmed in all but 2 patients by coronary angiography showing coronary stenosis with at least $75 \%$ narrowing in at least one vessel. CAD was identified in the other 2 patients by myocardial scintigraphy instead of coronary angiography because of renal dysfunction or contrast agent allergy. Clinical data from45 patients without CAD who had undergone CEA and were admitted during the same period were collected as control data. None of the 45 controls had any history of chest symptoms or any evidence of myocardial ischemia as assessed from both electrocardiography and transthoracic echocardiography. All patients underwent CEA for severe carotid stenosis or unstable plaque under general anesthesia with propofol. Surgical indications for carotid stenosis were as follows: linear stenosis $>70 \%$, area stenosis
$>90 \%$, and peak flow velocity in the internal carotid artery (ICA) $>200 \mathrm{~cm} / \mathrm{s}$ on transcranial color-coded sonography. Exclusion criteria were the presence of aphasia or physical deficits preventing the conduct of neurocognitive examinations. Patients who had previously undergone neurocognitive assessment were not included in this study, to avoid any risk of practice effect.

\section{Cognitive assessment}

The Japanese versions of Cognistat and the Frontal Assessment Battery (FAB) were adopted to assess cognitive functions [7-10]. Cognisant consists of 10 sub-components and severity of impairment in each cognitive domain was defined as follows: score $\geq 9$, normal; 8 , mild impairment; 7 , moderate impairment; $\leq 6$, severe impairment[8]. The FAB consists of 6 sub-components, and the following cutoff values for total score were applied: < 60 years old, 15; 60-69 years old, 13; $\geq 70$ years old, 11[11]. Cognitive decline was defined as both FAB total score below the cutoff and severe impairment in at least one domain of Cognistat.

\section{Magnetic resonance imaging (MRI)}

All patients underwent MRI on the same day or the day before the cognitive examination to assess white matter lesions of the brain. Deep White-Matter Hyperintensity (DWMH) and periventricular hyperintensity $(\mathrm{PVH})$ on T2-weighted imaging were classified using Fazekas grade [12]. Intracranial atherosclerosis was defined as $>50 \%$ stenosis of the ICA, middle cerebral artery, or anterior cerebral artery on MR angiography.

\section{Statistical analysis}

Age was represented as mean \pm standard deviation. Other data were represented as median and interquartile range, except where indicated otherwise. Statistical analysis was performed using the Wilcoxon rank-sum test for non-parametric data. Differences in binary variables were assessed using Pearson's chi-square test. Univariate analysis was used to evaluate associations between variables and cognitive decline. One-way analysis of variance was used to compare cognitive test scores among DWMH grades. Differences were considered significant for values of $\mathrm{p}<0.05$. All statistical analyses were performed using JMP Pro version 13.2software (SAS Institute, Cary, NC).

\section{Ethics}

The study was approved by the National Hospital Organization Kyushu Medical Center Ethical Review Board (18C194). 


\section{Results}

\section{Baseline characteristics}

Patients with CAD comprised 29 men and 7 women (angina pectoris, $n=17$; myocardial infarction, $n=10$;asymptomatic myocardial ischemia, $n=6$; silent myocardial infarction, $n=3$ ). Three patients had undergone coronary artery bypass grafting(CABG) alone before CEA, and 2 had undergone CABG and percutaneous coronary intervention (PCI) before CEA. Nine patients had undergone PCI alone before CEA and 1 had undergone PCI before and after CEA. Two patients had undergone CABG alone after CEA and 1 had undergone CABG and PCI after CEA. Four patients had undergone PCI alone after CEA. In CAD patients who had not undergone CABG nor PCI, coronary angiography showed 50-75\% stenosis in the left anterior descending branch with or without more than $75 \%$ narrowing in the other branches, with the exception of the 2 patients who did not undergo coronary angiography.

Table 1 shows that no significant differences between patients with and without CAD were noted in any baseline data except left carotid artery stenosis on examination. Left carotid stenosis was more severe in patients with CAD than in patients without CAD $(\mathrm{p}=0.0164)$. Most patients were receiving pharmacologic therapy for hypertension and dyslipidemia and were taking an antiplatelet agent. Brain infarction $>3 \mathrm{~cm}$ in diameter was observed in $19.4 \%$ of CAD patients and $22.2 \%$ of patients without CAD at the examination. Mean Fazekas DWMH grade and PVH grade did not differ between patients with and without CAD. Table 2 shows that no significant differences between patients with and without CAD were noted in symptomatic onset, operated side, postoperative complications, or intraoperative use of an internal shunt.

Table 1. Baseline demographic characteristics at the examination

\begin{tabular}{|c|c|c|c|}
\hline & With CAD $(n=36)$ & Without CAD $(n=45)$ & $P$ value \\
\hline Age (years) & $77.8 \pm 5.3$ & $75.7 \pm 6.5$ & 0.1224 \\
\hline Follow-up period (years) & $7.0[5.0,9.0]$ & $5.1[4.0,7.1]$ & 0.1174 \\
\hline Male (\%) & $29(80.6)$ & $37(82.2)$ & 0.8478 \\
\hline \multicolumn{4}{|l|}{ Medical history (\%) } \\
\hline Hypertension & $34(94.4)$ & $42(93.3)$ & 0.8364 \\
\hline Diabetes mellitus & $16(44.4)$ & $23(51.1)$ & 0.5507 \\
\hline Dyslipidemia & $35(97.2)$ & $40(88.9)$ & 0.1547 \\
\hline Chronic kidney disease & $11(30.6)$ & $7(15.6)$ & 0.1066 \\
\hline Atherosclerosis obliterans & $6(16.7)$ & $2(4.4)$ & 0.0669 \\
\hline Atrial fibrillation & $3(8.3)$ & $1(2.2)$ & 0.2072 \\
\hline Smoking history & $26(72.2)$ & $35(77.8)$ & 0.5645 \\
\hline Alcohol $>40$ g/day & $4(11.1)$ & $1(2.2)$ & 0.0986 \\
\hline Use of antiplatelet & $35(97.2)$ & $44(97.8)$ & 0.8728 \\
\hline Use of anticoagulant & $4(11.1)$ & $2(4.4)$ & 0.2549 \\
\hline Dementia & 0 & $3(6.7)$ & 0.1144 \\
\hline Brain infarction & $7(19.4)$ & $10(22.2)$ & 0.7603 \\
\hline \multicolumn{4}{|l|}{ CA stenosis at examination (\%) } \\
\hline Right & $41.8[0,54.5]$ & $47.0[0,60.4]$ & 0.3283 \\
\hline Left & $52.0[38.6,59.0]$ & $37.0[0,53.1]$ & 0.0164 \\
\hline Contralateral ICA occlusion (\%) & $1(2.8)$ & $1(2.2)$ & 0.8728 \\
\hline Intracranial atherosclerosis (\%) & $8(22.2)$ & $9(20.0)$ & 0.8072 \\
\hline DWMH grade & $1[1,2]$ & $1[0,1]$ & 0.1266 \\
\hline DWMH grade $\geq 2(\%)$ & $12(33.3)$ & $9(20.0)$ & 0.1736 \\
\hline PVH grade & $1[0,1.8]$ & $1[0,2]$ & 0.5956 \\
\hline $\mathrm{PVH}$ grade $\geq 2(\%)$ & $8(22.2)$ & $12(26.7)$ & 0.6448 \\
\hline
\end{tabular}

CAD, coronary artery disease; CA, carotid artery; ICA, internal carotid artery; DWMH, deep white matter hyperintensity; $\mathrm{PVH}$, periventricular hyperintensity 
Table 2.Perioperative demographic characteristics.

\begin{tabular}{llll}
\hline & With CAD (n=36) & $\begin{array}{l}\text { Without CAD } \\
(\mathrm{n}=45)\end{array}$ & P value \\
\hline Symptomatic onset (\%) & $18(50.0)$ & $32(71.1)$ & 0.0521 \\
Operated side (\%) & & & 0.1298 \\
$\quad$ Right & $23(63.9)$ & $21(46.7)$ & \\
$\quad$ Left & $9(25.0)$ & $21(46.7)$ & \\
$\quad$ Bilateral & $4(11.1)$ & $3(6.7)$ & \\
Hyperintensity on postoperative DWI (\%) & $3(8.3)$ & $6(13.3)$ & 0.4768 \\
Postoperative HP (\%) & 0 & $2(4.4)$ & 0.2003 \\
Postoperative HPS (\%) & 0 & $1(2.2)$ & 0.3681 \\
Use of internal shunt (\%) & $13(36.1)$ & $18(40.0)$ & 0.7205 \\
\hline
\end{tabular}

CAD, coronary artery disease; DWI, diffusion-weighted imaging; HP, hyperperfusion; HPS, hyperperfusion syndrome

\section{Cognitive assessment}

No significant difference in total Cognistat score or total FAB score was evident between patients with and without CAD. Scores for comprehension, memory, and conceptualization were significantly lower in patients with CAD than in patients without CAD $(\mathrm{p}=0.0274,0.0392$, and 0.0113 , respectively; Table 3). Cognitive decline, defined as FAB total score below the cutoff along with severe impairment in at least one domain on Cognistat, was seen in 8 patients with CAD (27.8\%), and in 4 patients without CAD (8.9\%).

In patients with $\mathrm{CAD}$, univariate analysis revealed that DWMH and chronic kidney disease (CKD) were associated with cognitive decline (odds ratio (OR) 32.2, 95\% confidence interval (CI) 3.20-323.66, $\mathrm{p}=0.0032$; OR 6.11, 95\%CI 1.13-33.19; $\mathrm{p}=0.0360$, respectively; Table 4). The association between DWMH and CKD was not evident $(\mathrm{p}=0.3061)$, and the association between DWMH and cognitive decline persisted after adjusting for CKD ( $\mathrm{p}=0.0004)$. Table 5 describes cognitive test scores and DWMH grades in patients with CAD. Total Cognistat score, total FAB score, scores for comprehension, similarity, programming, and sensitivity to interference declined as Fazekas DWMH grade increased. Univariate analysis showed no association between carotid stenosis and cognitive decline ( $\mathrm{p}=0.1739$ for right carotid stenosis; $\mathrm{p}=0.1954$ for left carotid stenosis).

\section{Discussion}

Cognitive decline was evident in $27.8 \%$ of patients with CAD a a mean of 7.2 years after CEA, and in $8.9 \%$ in patients without CAD at a mean of 6.1 years after CEA. We also found that comprehension, memory, and conceptualization were impaired compared to patients without CAD. DWMH and chronic kidney disease were risk factors or predictors for cognitive decline long after CEA. In particular, DWMH was associated with declines in several specific cognitive domains.

Carotid stenosis, CAD, and cognitive decline share modifiable cardiovascular risk factors such as obesity, diabetes, smoking, hypertension, physical inactivity, and dyslipidemia $[13,14]$. Our results indicated that cognitive decline long after CEA was more common in patients with CAD compared to patients without CAD. Left carotid artery stenosis at examination was more severe among CAD patients, suggesting that atherosclerosis might be more critical in patients with CAD than in those without. However, no significant differences were evident in intracranial atherosclerosis, DWMH, CKD, arteriosclerosis obliterans, or cardiovascular risk factors such as hypertension, diabetes, and dyslipidemia between patients with and without CAD. On the other hand, CAD appeared to be associated with cognitive decline. A study of 1101 patients with CAD aged $>65$ years showed the prevalence of cognitive impairment was $62 \%$ [15]. The presence of CAD accelerated cognitive decline as measured with the Mini-Mental State Examination by about 66\% in 118 patients with Alzheimer's or mixed dementia [16]. 
Table 3. Cognitive examination

\begin{tabular}{llll}
\hline & With CAD $(\mathrm{n}=36)$ & Without CAD $(\mathrm{n}=45)$ & P value \\
\hline Cognistat & $94[82,97.8]$ & $96[92,100]$ & 0.0966 \\
Total score & $10[9.3,10]$ & $10[10,10]$ & 0.5091 \\
Orientation & $10[6,10]$ & $10[8,10]$ & 0.3698 \\
Attention & $10[7,10]$ & $10[10,10]$ & 0.0274 \\
Comprehension & $11[8,11]$ & $11[8.5,11]$ & 0.2108 \\
Repetition & $10[10,10]$ & $10[9,10]$ & 0.5799 \\
Naming & $9[7.3,11]$ & $9[8,11]$ & 0.3634 \\
Construction & $7.5[6,9]$ & $8[7,10]$ & 0.0392 \\
Memory & $10[8.5,10]$ & $10[10,10]$ & 0.1122 \\
Calculation & $10[9,10]$ & $10[9,10]$ & 0.1551 \\
Similarity & $10[9,11]$ & $10[10,11]$ & 0.4356 \\
Judgment & $13[11,16]$ & & 0.2093 \\
FAB & $2[1,2]$ & $14[12,16]$ & 0.0113 \\
Total score & $2[1,2]$ & $2[2,2]$ & 0.1963 \\
Conceptualization & $2[1,3]$ & $2[1.5,3]$ & 0.7588 \\
Mental flexibility & $3[3,3]$ & $2[1,3]$ & 0.4302 \\
Programming & $2[1,3]$ & $3[3,3]$ & 0.4740 \\
Sensitivity to interference & $3[3,3]$ & $3[3,3]$ & 0.2743 \\
Inhibitory control & $12[9,16]$ & $12[12,16]$ & $0[0,1]$ \\
Environmental autonomy & $0[0,1]$ & $100[100,100]$ & 0.2074 \\
Education (years) & $100[100,100]$ & & 0.8132 \\
mRS at examination & & 0.8328 \\
Barthel index at examination & & & \\
\hline
\end{tabular}

CAD, coronary artery disease; FAB, frontal assessment battery; mRS, modified Rankin scale

Table 4. Univariate analysis for predictors of cognitive decline in patients with coronary artery disease.

\begin{tabular}{lll}
\hline Variable & Odds ratio $(95 \%$ Confidence interval) & P value \\
\hline Deep white matter hyperintensity & $32.2(3.20-323.66)$ & 0.0032 \\
Chronic kidney disease & $6.11(1.13-33.19)$ & 0.0360 \\
Age & $0.86(0.73-1.03)$ & 0.0720 \\
Education & $1.20(0.95-1.52)$ & 0.1235 \\
Alcohol & $4.33(0.50-37.26)$ & 0.1816 \\
Arteriosclerosis obliterans bypass & $2.78(0.38-20.50)$ & 0.3164 \\
Coronary artery bypass graft & $0.43(0.05-4.12)$ & 0.4631 \\
Male & $0.65(0.10-4.23)$ & 0.6541 \\
Current smoking & $1.53(0.24-9.95)$ & 0.6541 \\
Periventricular hyperintensity & $1.22(0.20-7.68)$ & 0.8305 \\
Left ventricular ejection fraction & $0.93(0.78-1.12)$ & 0.8315 \\
Smoking history & $1.20(0.20-7.25)$ & 0.8425 \\
Percutaneous coronary intervention & $1.00(0.21-4.81)$ & 1.0000 \\
\hline
\end{tabular}


Table 5. Cognitive examination by deep white matter hyperintensity in patients with coronary artery disease.

\begin{tabular}{|c|c|c|c|c|c|}
\hline & \multicolumn{4}{|c|}{ Deep white matter hyperintensity grade } & \multirow{2}{*}{$P$ value } \\
\hline & $0(n=7)$ & $1(n=17)$ & $2(n=4)$ & $3(n=8)$ & \\
\hline \multicolumn{6}{|l|}{ Cognistat } \\
\hline Total score & $97[95,99]$ & $95[88.5,98.5]$ & $76[71.5,89.5]$ & $84[68.75,94]$ & 0.0086 \\
\hline Orientation & $10[10,10]$ & $10[9.5,10]$ & $9.5[6,10]$ & $10[6.75,10]$ & 0.3257 \\
\hline Attention & $10[6,10]$ & $10[8,10]$ & $6[3.75,9]$ & $8[1.5,10]$ & 0.2782 \\
\hline Comprehension & $10[10,10]$ & $10[8.5,10]$ & $7[4.75,9.25]$ & $8.5[4,10]$ & 0.0320 \\
\hline Repetition & $11[10,11]$ & $11[8,11]$ & $7[6.25,10]$ & $10.5[6,11]$ & 0.2264 \\
\hline Naming & $10[10,10]$ & $10[10,10]$ & $9[7.5,9.75]$ & $10[9.25,10]$ & 0.1697 \\
\hline Construction & $9[8,11]$ & $9[8,11]$ & $7[6,8.75]$ & $7.5[6,10.25]$ & 0.0581 \\
\hline Memory & $8[5,9]$ & $8[6,9]$ & $8[6.25,9]$ & $6.5[5.25,8]$ & 0.6135 \\
\hline Calculation & $10[10,10]$ & $10[10,10]$ & $9[6.5,10]$ & $9[6.5,10]$ & 0.1334 \\
\hline Similarity & $10[9,10]$ & $10[9,10]$ & $7.5[6.25,9.5]$ & $9[8.25,10.75]$ & 0.0418 \\
\hline Judgment & $10[9,12]$ & $10[10,11]$ & $9.5[9,10]$ & $9.5[9,11]$ & 0.5093 \\
\hline \multicolumn{6}{|l|}{ Frontal assessment battery } \\
\hline Total score & $14[13,17]$ & $15[12.5,16]$ & $9.5[8.25,13]$ & $10.5[8.5,11.75]$ & 0.0002 \\
\hline Conceptualization & $2[2,2]$ & $2[2,2]$ & $0[0,1.5]$ & $2[1,2]$ & 0.0059 \\
\hline Mental flexibility & $2[2,3]$ & $2[1,2]$ & $2[1.25,2]$ & $1.5[0.25,2.75]$ & 0.1820 \\
\hline Programming & $3[1,3]$ & $3[2,3]$ & $1.5[1,2]$ & $1[0.25,2.75]$ & 0.0311 \\
\hline Sensitivity to interference & $3[3,3]$ & $3[3,3]$ & $3[0.75,3]$ & $2[0.25,3]$ & 0.0223 \\
\hline Inhibitory control & $3[2,3]$ & $2[1,3]$ & $1.5[0.25,2]$ & $1[1,2.75]$ & 0.0880 \\
\hline Environmental autonomy & $3[3,3]$ & $3[3,3]$ & $3[3,3]$ & $3[3,3]$ & 0.3309 \\
\hline
\end{tabular}

A recent meta-analysis of 10 prospective cohort studies representing 24,801 persons indicated that CAD was associated with increased risk of cognitive decline $(\mathrm{OR}=1.45,95 \% \mathrm{CI}=1.21-$ $1.74, \mathrm{p}<0.001)[17]$.

We found that comprehension, memory, and conceptualization were significantly lower in patients with CAD compared to patients without CAD. Attention, memory, executive function, visuospatial orientation, psychomotor speed, and fluency improved shortly after CEA [2,18-21], while language, working memory, and global cognition deteriorated [1,22-25]. These were all short-term changes, and long-term changes in cognitive domains after CEA are still unknown. On the other hand, global cognition, verbal memory, and executive function declined in CAD patients who were cognitively intact at baseline after a mean follow-up of 6.9 years [26]. Working memory, processing speed, cognitive inhibition, flexibility, and long-term verbal memory were impaired even in patients with stable CAD compared to age-matched old health controls [27].

Several studies have reported various risk factors of cognitive decline short after CEA. Age, education, intelligent quality, brain atrophy, preoperative symptoms, and white mat- ter hyperintensity were associated with cognitive changes at 1-6 months after CEA $[22,28,29]$, but factors relating to long-term cognitive changes after CEA are still unclear. Post-hoc meta-regression analyses showed that the association between CAD and cognitive decline was unchanged after adjusting for risk factors such as diabetes, hypertension, and dyslipidemia, suggesting that this association cannot be solely explained by cardiovascular risk factors [17]. A systematic review identified CABG, apolipoprotein E4, left ventricular ejection fraction, medication use, and hormones and biomarkers as predictors of cognitive decline in patients with CAD [30].Conversely, a prospective longitudinal study found no significant differences in long-term cognitive outcomes among CAD patients treated surgically with on-pump CABG $(n=152)$ or off-pump CABG $(n=75))$ or non-surgically $(n=99)[6]$. This is consistent with our results from univariate analysis.

Our results indicated that DWMH was significantly associated with cognitive decline and DWMH grade was associated with declines in several cognitive domains among CAD patients long after CEA. Cerebral white matter hyperintensity (WMH) has been shown to be strongly associated with an increased risk of cognitive impairment [31]. Community-based epidemiologic 
studies have revealed that $\mathrm{WMH}$ and silent brain infarct (SBI) were associated with cerebrovascular risk factors and cognitive decline [32-35]. One study reported that CEA prevented increases in WMH at 1 year after surgery in 14 patients who were already cognitively impaired preoperatively [2]. Another study revealed that $\mathrm{WMH}$ was one of the differences between cognitive responders and non-responders at 1 year after CEA, but $\mathrm{WMH}$ was not independent associated with cognitive decline in multivariate analysis [22].On the other hand, a cross-sectional study showed that cerebral white matter microstructural integrity was associated with executive function impairment in 49 patients with CAD [36]. Longitudinal studies demonstrated that progression of WMH grade was associated with declines in overall cognitive function and several specific domains [34, 35], but the associations between $\mathrm{CAD}$ and cognitive decline persisted after adjusting for both baseline levels and changes in $\mathrm{WMH}$ and SBI, indicating that these associations are not fully explained by MRI-measured WMH and SBI [26].

One strength of this study was a long follow-up. We evaluated neurocognitive functions in patients with CAD at a mean of 7.2 years after CEA, using two representative cognitive batteries. This study has several limitations. First, the study was cross-sectional in design. When the neurocognitive function starts to decline and the influence of progressive DWMH changes over time on cognitive decline in CAD patients are unknown. To clarify sequential changes in neurocognitive function, longitudinal studies are needed. Second, the sample size was relatively small. We could not perform multivariate analysis for predictors of cognitive decline.

\section{Conclusions}

Our findings indicate that long-term neurocognitive outcomes in several cognitive domains were inferior in patients with CAD compared to those in patients without CAD.

\section{References}

1. Lal BK, Younes M, Cruz G, Kapadia I, Jamil Z, Pappas PJ (2011) Cognitive changes after surgery vs stenting for carotid artery stenosis. J Vasc Surg 54:691-698.

2 . Dempsey RJ, Jackson DC, Wilbrand SM, Mitchell CC, Berman SE, Johnson SC, et al. (2018) The Preservation of Cognition 1 Year After Carotid Endarterectomy in Patients With Prior Cognitive Decline. Neurosurgery 82:322-328.

3. Lal BK (2007) Cognitive function after carotid artery revascularization. Vasc Endovascular Surg 41:5-13.

4. Singh-Manoux A, Sabia S, Lajnef M, Ferrie JE, Nabi H, Britton AR, et al. (2008) History of coronary heart disease and cognitive performance in midlife: the Whitehall II study. Eur Heart J 29:2100-2107.

5. Haring B, Leng X, Robinson J, Johnson KC, Jackson RD, Beyth $\mathrm{R}$, et al. (2013) Cardiovascular disease and cognitive decline in postmenopausal women: results from the Women's Health Initiative Memory Study. J Am Heart Assoc 2:e000369.

6. Selnes OA, Grega MA, Bailey MM, Pham LD, Zeger SL, Baumgartner WA, et al. (2009) Do management strategies for coronary artery disease influence 6-year cognitive outcomes? Ann Thorac Surg 88:445-454.

7. Kiernan RJ, Mueller J, Langston JW, Van Dyke C (1987) The Neurobehavioral Cognitive Status Examination: a brief but quantitative approach to cognitive assessment. Ann Intern Med 107:481-485.

8 Matsuda O, Nakatani M (2004) The manuals of the Japanese version of the neurobehavioral cognitive status examination. Tokyo, World Plan.

9 Dubois B, Slachevsky A, Litvan I, Pillon B (2000) The FAB a Frontal Assessment Battery at bedside. Neurology 55:1621-1626.

10. Kugo A, Terada S, Ata T, Ido Y, Kado Y, Ishihara T, et al. (2007) Japanese version of the Frontal Assessment Battery for dementia. Psychiatry Res 153:69-75. 
11. Terada T, Obi T, Sugiura A, Yamazaki K, Mizoguchi K (2009) Effect of aging on the Frontal Assessment Battery (FAB). Jpn J Neuropsychol 25:51-56.

12. Fazekas F, Chawluk JB, Alavi A, Hurtig HI, Zimmerman RA (1987) MR signal abnormalities at $1.5 \mathrm{~T}$ in Alzheimer's dementia and normal aging. AJR Am J Roentgenol 149:351-356.

13. Justin BN, Turek M, Hakim AM (2013) Heart disease as a risk factor for dementia. Clin Epidemiol 5:135-145.

14. Deckers K, van Boxtel MPJ, Schiepers OJG, de Vugt M, Muñoz Sánchez JL, Anstey KJ, et al. (2015) Target risk factors for dementia prevention: a systematic review and Delphi consensus study on the evidence from observational studies. Int J Geriatr Psychiatry 30:234-246.

15. Shavelle RM, Paculdo DR, Strauss DJ, Kush SJ (2009) Cognitive impairment and mortality in the Cardiovascular Health Study. J Insur Med N Y N 41:110-116.

16. Bleckwenn M, Kleineidam L, Wagner M, Jessen F, Weyerer S, Werle J, et al. (2017)Impact of coronary heart disease on cognitive decline in Alzheimer's disease: a prospective longitudinal cohort study in primary care. Br J Gen Pr 67:e111-e117.

17. Deckers K, Schievink SHJ, Rodriquez MMF, van Oostenbrugge RJ, van Boxtel MPJ, Verhey FRJ, et al. (2017) Coronary heart disease and risk for cognitive impairment or dementia: Systematic review and meta-analysis. PLoS ONE 12.

18. Usman R, Jamil M, Haq IU, Memon AA (2016) Neurocognitive Improvement in Patients Undergoing Carotid Endarterectomy for Atherosclerotic Occlusive Carotid Artery Disease. Ann Vasc Dis 9:307-311.

19. Kougias P, Collins R, Pastorek N, Sharath S, Barshes NR, McCulloch K, et al. (2015) Comparison of domain-specific cognitive function after carotid endarterectomy and stenting. J Vasc Surg 62:355-361.

20. Fearn SJ, Hutchinson S, Riding G, Hill-Wilson G, Wesnes K, McCollum CN (2003) Carotid endarterectomy improves cognitive function in patients with exhausted cerebrovascular reserve. Eur J VascEndovasc Surg 26:529-536.
21. Bossema ER, Brand N, Moll FL, Ackerstaff RGA, van Doornen LJP (2005) Does carotid endarterectomy improve cognitive functioning? J Vasc Surg 41:775-781.

22. Casas-Hernanz L, Garolera M, Badenes D, Quintana S, Millán S, Calzado N, et al. (2017) Neuropsychological Outcome One Year after Carotid Revascularization: A before-and-after Study. Vasc Spec Int 33:146-155.

23. Brand N, Bossema ER, OmmenMv M van, Moll FL, Ackerstaff RGA (2004) Left or right carotid endarterectomy in patients with atherosclerotic disease: ipsilateral effects on cognition? Brain Cogn 54:117-123.

24. Feliziani FT, Polidori MC, De Rango P, Mangialasche F, Monastero R, Ercolani S, et al. (2010) Cognitive performance in elderly patients undergoing carotid endarterectomy or carotid artery stenting: a twelve-month follow-up study. Cerebrovasc Dis 30:244-251.

25. Ogasawara K, Yamadate K, Kobayashi M, Endo H, Fukuda T, Yoshida K, et al. (2005) Postoperative cerebral hyperperfusion associated with impaired cognitive function in patients undergoing carotid endarterectomy. J Neurosurg 102:38-44.

26. Zheng L, Mack WJ, Chui HC, Heflin L, Mungas D, Reed B, et al. (2012) Coronary Artery Disease is Associated with Cognitive Decline Independent of MRI Changes in Cognitively Normal Elderly. J Am Geriatr Soc 60:499-504.

27. Gayda M, Gremeaux V, Bherer L, Juneau M, Drigny J, Dupuy O, et al. (2017) Cognitive function in patients with stable coronary heart disease: Related cerebrovascular and cardiovascular responses. PloS One 12:e183791.

28. Carta MG, Lecca ME, Saba L, Sanfilippo R, Pintus E, Cadoni $M$, et al. (2015) Patients with carotid atherosclerosis who underwent or did not undergo carotid endarterectomy: outcome on mood, cognition, and quality of life. BMC Psychiatry 15:277.

29. Pearson S, Maddern G, Fitridge R (2003) Cognitive performance in patients after carotid endarterectomy. J Vasc Surg 38:1248-1252.

30. Burkauskas J, Lang P, Bunevičius A, Neverauskas J, BučiūtèJankauskienè M, Mickuvienè N (2018) Cognitive function in patients with coronary artery disease: A literature review. J Int Med Res 46:4019-4031. 
31. Zhou G, Ren S, Chen N, Duan L, Zhang Z, Fang S, et al. (2008) Cerebral white matter lesions and cognitive function in a nondemented Chinese veteran cohort. J Int Med Res 36:115-122.

32. Debette S, Beiser A, DeCarli C, Au R, Himali JJ, Kelly-Hayes M, et al. (2010) Association of MRI markers of vascular brain injury with incident stroke, mild cognitive impairment, dementia, and mortality: the Framingham Offspring Study. Stroke 41:600606.

33. Seshadri S, Wolf PA, Beiser A, Elias MF, Au R, Kase CS, et al. (2004) Stroke risk profile, brain volume, and cognitive function: the Framingham Offspring Study. Neurology 63:1591-1599.

34. van Dijk EJ, Prins ND, Vrooman HA, Hofman A, Koudstaal PJ, et al. (2008) Progression of cerebral small vessel disease in relation to risk factors and cognitive consequences: Rotterdam Scan Study. Stroke 39: 2712-2719.

35. Longstreth WT, Arnold AM, Beauchamp NJ, Manolio TA, et al. (2005) Incidence, manifestations, and predictors of worsening white matter on serial cranial magnetic resonance imaging in the elderly: the Cardiovascular Health Study. Stroke 36:56-61.

36. Santiago C, Herrmann N, Swardfager W, Saleem M, Oh PI, Black SE, et al. (2015) White Matter Microstructural Integrity Is Associated with Executive Function and Processing Speed in Older Adults with Coronary Artery Disease. Am J Geriatr Psychiatry Off J Am Assoc Geriatr Psychiatry 23:754-763.

Submit your manuscript to a JScholar journal and benefit from:

ฯ Convenient online submission

ฯ Rigorous peer review

ฯ Immediate publication on acceptance

ๆ Open access: articles freely available online

I High visibility within the field

ब Better discount for your subsequent articles

Submit your manuscript at http://www.jscholaronline.org/submit-manuscript.php 